\title{
Conservation of coral reef biodiversity: a comparison of reserve selection procedures for corals and fishes
}

\author{
Maria Beger*, Geoffrey P. Jones, Philip L. Munday \\ School of Marine Biology and Aquaculture, Centre for Coral Reef Biodiversity, James Cook University, Townsville, QLD 4811, Australia
}

Received 11 January 2002; received in revised form 31 May 2002; accepted 17 June 2002

\begin{abstract}
A range of different biodiversity-based selection methods for nature reserves has been tested for terrestrial environments, including those based on diversity hotspots, endemicity hotspots and complementarity. In this study, we investigate the utility of these approaches for a coral reef embayment. We compare coral and fish species richness in a random accumulation of reserve sites with (a) hotspots analysis, (b) stratified selection of hotspots, and (c) complementarity. Cumulative species-site curves indicated that complementarity maximized the rate of accumulation of species of both corals and fishes in reserves, while the hotspot approach performed moderately well. An equivalent number of reserve sites supported a greater proportion of the coral biodiversity when compared to fishes, reflecting the broader distribution of corals. Our results indicate that when choosing an indicator group as a proxy for representing overall diversity in a reserve network, the group with the greatest heterogeneity will provide the best results. Our findings also show that although a modest number of protected sites $(20 \%)$ will incorporate much of the local diversity $(>75 \%)$, species-specific approaches must be incorporated to target rare species.

(C) 2002 Elsevier Science Ltd. All rights reserved.

Keywords: Marine protected areas; Coral reef conservation; Biodiversity; Reserve selection; Complementarity
\end{abstract}

\section{Introduction}

Marine protected areas are a widely recognised means for both fisheries management and the conservation of biodiversity (Salm, 1984; Roberts and Polunin, 1991; Jones et al., 1992; Allison et al., 1998; Salm et al., 2000; Hixon et al., 2001). Maximising the biodiversity in protected areas has not played a central role in selecting sites for marine reserves, particularly in the Indo-Pacific region. The role of protected areas in conserving and enhancing fish stocks has received far greater attention, particularly in coral reef environments where increases of stocks inside protected areas have been demonstrated at several locations (Roberts et al., 2001). More often than not, reserve selection has been opportunistic, depending on areas becoming available for conservation, political circumstances and local goodwill (Hatcher et al., 1989; Courtney and White, 2000; Salm et al., 2000). This process is likely to be sub-optimal from the point of view of protecting as many species as

\footnotetext{
* Corresponding author.

E-mail address: maria.beger@jcu.edu.au (M. Beger).
}

possible (Prendergast et al., 1999) and maintaining ecosystem services (Duarte, 2000).

In conservation planning for terrestrial systems, theory relating to biodiversity has played an important role (Prendergast et al., 1999). Different algorithms for establishing priorities in the selection of reserve sites have been developed and tested (Margules and Nicholls, 1988; Pressey et al., 1993; Williams et al., 1996; Howard et al., 1998; Prendergast et al., 1999). Selection approaches have included analysis of bio-geographical distributions (Turpie et al., 2000), hotspots (sites with maximum counts of all or rare species), complementarity of species richness or rarity (Margules and Nicholls, 1988; Vane-Wright et al., 1991) and irreplaceability value (Ferrier et al., 2000; Pressey and Taffs, 2001). These usually perform better than randomly choosing reserve sites. When combinations of these procedures have been compared, complementaritybased methods for both richness and rarity usually minimise the number of sites necessary to represent the greatest number of species (Pressey et al., 1993; Williams et al., 1996; Reyers et al., 2000; Turpie et al., 2000), while selecting hotspots of species richness does 
not usually provide the best representation of all species (Gaston, 1996; Williams et al., 1996; Reid, 1998).

There have been few attempts to establish the best procedures for selecting sites to protect marine biodiversity (Hixon et al., 2001). The best biodiversity-based approach to selecting reserve sites in marine and terrestrial environments may differ, due to differences in the scales of spatial variation in biodiversity (Steele, 1985; May, 1994; Hixon et al., 2001). Recently, some methods of prioritisation have been applied to marine environments (Turpie et al., 2000; Day et al., in press; Leslie et al., in press) but there has been no systematic comparison of the reserve selection approaches.

The use of indicator groups in reserve selection may achieve a reduction of data acquisition effort and cost (Williams and Gaston, 1994). This is particularly applicable when taxa exhibit a high degree of congruency, and spatial overlap of hotspots, endemicity and complementarity. An understanding of these biodiversity patterns can improve the selection process (Gaston, 1996; Howard et al., 1998; Reyers et al., 2000; Moritz et al., 2001). While there is not always a great deal of concordance between different terrestrial taxa (Prendergast et al., 1993; Gaston et al., 1995), indicator groups have proven useful in certain cases (Balmford, 1998; Howard et al., 1998; Reyers et al., 2000; Moritz et al., 2001). Two studies have suggested that indicator taxa can be useful in marine temperate systems (Ward et al., 1999; Gladstone, 2002), but the approach has not been tested for coral reefs.

The application of biodiversity approaches requires detailed species lists from multiple locations (Gaston and Williams, 1996). In practice, this data is usually restricted to a few well-described taxa that have been described to an appropriate taxonomic level (Holthus, 1994; Werner and Allen, 1998). The cost and effort for such surveys are often too high for local organisations to afford, particularly for the developing countries in the global centre of coral reef biodiversity. However, a marine conservation network based on a few key taxa may capture a substantial part of overall biodiversity (Ward et al., 1999; Gladstone, 2002). This may be especially true on coral reefs where there is often a correlation between fishes, coral and reef invertebrate diversity on large spatial scales (Bellwood and Hughes, 2001). However, the degree to which corals can be used as a surrogate for fishes and vice versa is unknown for local marine reserve networks.

In this study, we apply different selection methods for selecting sites that maximise the biodiversity of fishes and corals in protected areas for a tropical embayment (Kimbe Bay) on the island of New Britain, Papua New Guinea. Kimbe Bay is recognised as an area of exceptional coral reef biodiversity with a total of 837 species of fishes and 347 species of coral recorded (Munday, 2000). We use a data set on species diversity of fishes and corals from 35 isolated reefs to answer the following questions: (1) What step-wise selection procedure provides the greatest accumulation of species in protected areas, for a given area of protection? We compare a random choice of reserve sites, with (a) hotspots analysis (including both species richness and rarity hotspots), (b) stratified hotspot selection within bio-geographical zones and (c) complementarity. (2) Does the best selection procedure apply to both fishes and corals? (3) Is there a general concordance between the preferred sites selected for fishes and corals? Finally, (4) if the goal is to provide the best level of protection for both fishes and corals, which taxon serves as the best surrogate for the other?

\section{Methods}

\subsection{The database}

The database for this study was compiled during a biodiversity assessment for Kimbe Bay, New Britain $\left(150^{\circ} 15^{\prime} \mathrm{E}, 5^{\circ} 15^{\prime} \mathrm{S}\right)$, Papua New Guinea, carried out in 1994 (Allen and Munday, 1994; Holthus, 1994; Holthus and Maragos, 1994; Maragos, 1994). Kimbe Bay is located within the Indo-Pacific centre of tropical marine biodiversity and has a low level of anthropogenic impacts (Munday, 2000). Presence-absence species counts from timed swims were available for hard corals and fishes at 35 sites, which are largely discrete coral reef platforms and pinnacles separated by deep water (Table 1).

All timed swim surveys took between 60 and $80 \mathrm{~min}$. During this time, the recorder began at a maximum depth of $25 \mathrm{~m}$, swimming back and forth along a depth contour while slowly moving into shallower water. The survey ended at the reef crest. The survey report lacked information on selection criteria for the reefs assessed, and how well one survey represented their total size (Allen and Munday, 1994; Holthus, 1994; Holthus and Maragos, 1994). However, based on the idea that each survey covered a similar area in the given time, we assume that the area of each site was roughly the same. All species of fishes and corals for which reliable site records were available were included in the analysis. For this study, we refer to the added number of coral and fish species as 'all species'.

\subsection{Species distribution}

Variation in species distributions are likely to have a major effect on area selection; and the incorporation of bio-geographic patterns have been recommended for large-scale reserve selection (Turpie et al., 2000). In order to verify whether inclusion of such information improves the reserve selection procedures we applied Multi-Dimensional Scaling (MDS) multivariate analysis to fishes and corals. The detected spatial patterns of community structure defined eco-habitats for the bio- 
Table 1

List of sites and species in Kimbe Bay

\begin{tabular}{|c|c|c|c|c|c|c|c|c|}
\hline \multirow[t]{2}{*}{ Site } & \multirow[t]{2}{*}{ Name } & \multirow[t]{2}{*}{ Region } & \multicolumn{3}{|c|}{ Number of species } & \multicolumn{3}{|c|}{ Number of rare species } \\
\hline & & & All & Fish & Coral & All & Fish & Coral \\
\hline $\mathrm{J} 26$ & Restorff Island & $\mathrm{OCZ}$ & 323 & 166 & 157 & 26 & 17 & 9 \\
\hline J29 & Kimbe Island south & $\mathrm{OZ}$ & 287 & 164 & 123 & 18 & 9 & 9 \\
\hline $\mathrm{J} 34$ & Wulai Reef & $\mathrm{OZ}$ & 280 & 183 & 97 & 10 & 8 & 2 \\
\hline $\mathbf{J} 22$ & Cape Heussner & $\mathrm{OZ}$ & 279 & 184 & 95 & 28 & 23 & 5 \\
\hline $\mathrm{J} 32$ & Wulai shelter & $\mathrm{OZ}$ & 273 & 145 & 128 & 7 & 3 & 4 \\
\hline J37 & Hogu Reef exposed & $\mathrm{OZ}$ & 271 & 179 & 92 & 6 & 6 & 0 \\
\hline $\mathrm{J} 14$ & Beacon Reef & $\mathrm{OZ}$ & 268 & 173 & 95 & 11 & 4 & 7 \\
\hline $\mathrm{J} 18$ & Coast south & $\mathrm{OCZ}$ & 263 & 133 & 130 & 9 & 3 & 4 \\
\hline J39 & Palanga coast & $\mathrm{OCZ}$ & 261 & 148 & 113 & 7 & 2 & 5 \\
\hline $\mathrm{J} 10$ & Big Mulumalu Island & $\mathrm{OCZ}$ & 259 & 184 & 75 & 15 & 12 & 3 \\
\hline $\mathrm{J} 36$ & Hogu Lagoon & $\mathrm{OCZ}$ & 252 & 151 & 101 & 6 & 2 & 4 \\
\hline $\mathbf{J} 25$ & Tele Reef & $\mathrm{OZ}$ & 248 & 142 & 106 & 8 & 6 & 2 \\
\hline J8 & Erna Reef right & $\mathrm{OZ}$ & 246 & 144 & 102 & 17 & 12 & 5 \\
\hline J31 & Oto Reef & $\mathrm{OZ}$ & 244 & 139 & 105 & 6 & 5 & 1 \\
\hline J9 & Erna Reef left & $\mathrm{OZ}$ & 241 & 171 & 70 & 3 & 3 & 0 \\
\hline $\mathrm{J} 38$ & Kilu coast & $\mathrm{OCZ}$ & 240 & 155 & 85 & 7 & 3 & 4 \\
\hline $\mathrm{J} 12$ & Paluma Reef & $\mathrm{OCZ}$ & 239 & 134 & 105 & 6 & 1 & 5 \\
\hline $\mathrm{J} 30$ & Kimbe Island north & $\mathrm{OZ}$ & 239 & 148 & 91 & 10 & 7 & 3 \\
\hline $\mathrm{J} 35$ & Hogu Reef west & $\mathrm{OZ}$ & 235 & 150 & 85 & 11 & 8 & 3 \\
\hline $\mathrm{J} 23$ & Island Heussner & $\mathrm{OZ}$ & 233 & 151 & 82 & 11 & 9 & 2 \\
\hline $\mathrm{J} 3$ & Lemu Reef & $\mathrm{OZ}$ & 231 & 123 & 108 & 9 & 5 & 4 \\
\hline J19 & Fish Reef & OCZ & 224 & 137 & 87 & 4 & 1 & 3 \\
\hline $\mathrm{J} 17$ & Numondo Reef & $\mathrm{SCZ}$ & 223 & 129 & 94 & 25 & 23 & 2 \\
\hline $\mathrm{J} 21$ & Moewen Reef & $\mathrm{OCZ}$ & 221 & 131 & 90 & 10 & 5 & 5 \\
\hline $\mathrm{J} 15$ & Coast North & $\mathrm{OCZ}$ & 220 & 113 & 107 & 4 & 2 & 2 \\
\hline $\mathrm{J} 24$ & Coast Heussner & $\mathrm{OCZ}$ & 214 & 131 & 83 & 6 & 5 & 1 \\
\hline J6 & Garua Island coast & SCZ & 210 & 136 & 74 & 16 & 12 & 4 \\
\hline $\mathrm{J} 33$ & Wulai shelter south & $\mathrm{OZ}$ & 209 & 130 & 79 & 6 & 2 & 4 \\
\hline $\mathrm{J} 27$ & Garua mud islands & SCZ & 205 & 108 & 97 & 5 & 4 & 1 \\
\hline $\mathrm{J} 20$ & Hoskins coast & $\mathrm{OCZ}$ & 198 & 130 & 68 & 7 & 6 & 1 \\
\hline $\mathrm{J} 13$ & Deception Point & $\mathrm{OCZ}$ & 192 & 144 & 48 & 17 & 13 & 4 \\
\hline $\mathrm{J} 28$ & Garua south bank & $\mathrm{OCZ}$ & 190 & 119 & 71 & 0 & 0 & 0 \\
\hline $\mathrm{J} 11$ & Coast near Garua & $\mathrm{OCZ}$ & 183 & 119 & 64 & 5 & 3 & 2 \\
\hline $\mathrm{J} 16$ & Kimbe coast & $\mathrm{SCZ}$ & 178 & 116 & 62 & 12 & 11 & 1 \\
\hline J5 & Schaumann Island & SCZ & 128 & 83 & 45 & 9 & 8 & 1 \\
\hline
\end{tabular}

OZ, outer zone; SCZ, sheltered coastal zone; OCZ, outer coastal zone. Rare species occur at no more than two sites. Site codes are reef identifiers from Holthus (1994).

geographical selection method. In this study, we used the term eco-habitats to describe a spatially distinct group of reefs that exhibit a distinct community structure owing to different environmental factors such as proximity to shore, water exchange, relief and reef community structure. The analysis was carried out for both coral and fishes. We also assessed concordance patterns in species distribution by testing the similarity of fish and coral data based on a Bray-Curtis similarity matrix using the RELATE programme of PRIMER (PRIMER, 2001).

\subsection{Selection procedures}

Selection methods were compared in their efficacy and suitability by species-area accumulation curves. Since our sites correspond to an equal sampling area, those curves plot the accumulated number of sites by the cumulative species percentage. Selection methods established the order in which sites were ranked. Species-site number accumulation curves were determined by the six selection procedures listed below.

\subsubsection{Random $(\mathrm{n}=6)$}

Random selections of sites without replacement were made and the accumulative species count calculated as a mean of six repeats (precision $P=0.007$ ).

\subsubsection{Hotspots (richness)}

Sites were selected in decreasing order of total species count for each taxon until all sites were included. The accumulative species number was calculated as sites were added. Sites with the same number of species were added in random order. In our data set, such sites had low species numbers, and their order had little impact on the overall shape of the resulting species accumulation curve. 


\subsubsection{Hotspots (rarity)}

Sites were selected in decreasing order of the species present at each site that occurred twice or less within the entire data set (rarity threshold $T=2$ ), using the reserve selection software WORLDMAP (Williams, 2000).

\subsubsection{Hotspots (three eco-habitats-richness)}

Areas were selected by alternating between sites belonging to each eco-habitat identified in the species distribution analysis. Within each subset, sites were selected in decreasing order of total species count. The first site was chosen by selecting the site with the highest total species number from all subsets, followed by the site with the higher species number from the two remaining subsets. This predetermined order of subsets was maintained for the entire calculation.

\subsubsection{Complementarity (rarity)}

Sites were ranked for maximum complementary rarity $(T=2)$ by a progressive rarity algorithm in WORLDMAP; for details of the algorithm, see Williams (2000) and Williams et al. (2000). If there was an alternative set of sites that would achieve the same conservation goal (i.e. there was a tie between sites), the algorithm selected the site with more rare species.

\subsubsection{Complementarity (richness)}

Areas were chosen by selecting for maximum complementary richness at each step. This was calculated by a simple greedy algorithm in WORLDMAP, based on Kirkpatrick (1993) and Vane-Wright et al. (1991) in Williams (2000).

\subsection{Congruency and representativeness}

The area required to represent a large number of species from a range of taxa depends on the degree of congruency among the different groups. In order to establish this for fishes and corals, we first compared the spatial overlap in species richness per site using Pearson Correlation coefficients (Howard et al., 1998). Since the selection process depends largely on rare species, we then correlated total species richness and number of rare species per site for fishes and corals using the same method.

The representativeness of a reserve network selected by one indicator group depends on how the distribution of the indicator taxon overlaps with other taxa (Williams et al., 2000). To determine representativeness of selections based on indicator or representative taxa, areas were chosen for each group (fishes, corals and all species) using the complementarity (richness) approach. The order of sites derived was then applied to the remaining taxa and plotted on a graph relating the cumulative species inclusion to the number of sites chosen.

\section{Results}

\subsection{Patterns in species distribution}

The inclusion of species in reserves as a function of the area coming under protection partially depends on large-scale patterns in species distributions or heterogeneity in community structure within the area of interest. Multi-dimensional scaling analysis indicated that there was greater spatial heterogeneity in the fish communities on the 35 reefs compared with the corals. Three fish communities were identified (Fig. 1a), which corresponded with a gradient from sheltered coastal reefs to offshore reefs (Fig. 2, Table 1). In contrast, the coral communities were largely homogeneous, except for a small number of sites with unique species composition (Fig. 1b). Hence, on the scale examined there was not a close correlation between fish and coral assemblages $(r=0.345, P=0.3)$, at least in terms of presenceabsence data. The three habitat systems identified by the fish assemblages were used as a basis for a bio-geographically stratified hotspot approach.

\subsection{Comparison of site selection procedures}

The complementarity-based approaches (based on either richness and rarity) consistently scored the greatest cumulative representation of species in protected areas during the step-wise addition of new reserve sites (Fig. 3). The two complementarity-based methods performed in a similar manner and always achieved higher accumulative species richness than the other methods, regardless of whether the focus was on fishes, corals or both taxa combined. The random addition of new sites was on average the least efficient method. The three hotspot methods scored within an intermediate range between these extremes, although choice of rarity hotspots consistently included more species than richness hotspots. The hotspots (richness) approach for all species and fishes was relatively efficient for low numbers of reserves, but with increasing numbers of sites added, it eventually performed no better than choosing sites on a random basis.

The species accumulation curves differed between fishes and corals for all methods used. Corals displayed the highest initial species inclusion, resulting in higher species percentages protected for fewer sites (Fig. 4). In contrast, fish curves start at lower species percentages and climb gradually, lying below the other curves for all methods. This reflects the greater regional occurrence of coral species at any one site and their broader distribution across the bay. The differences between fishes and corals are most apparent for the hotspots (richness) method and most similar for the best performing complementarity method. When both fishes and corals are combined the curve more closely resembles the pattern for fishes only, which can be attributed to the greater species richness of fishes. 
(a)

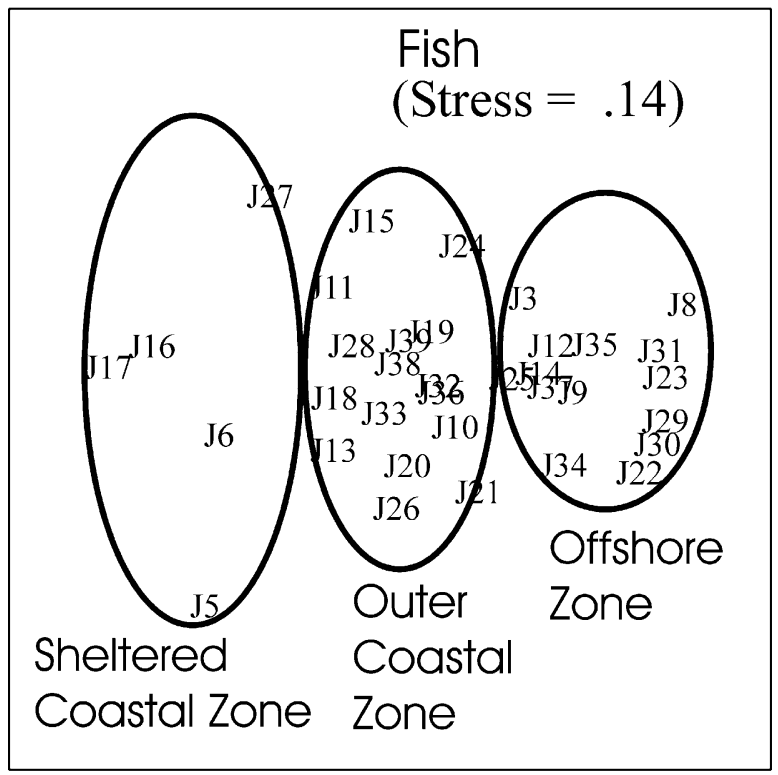

(b)

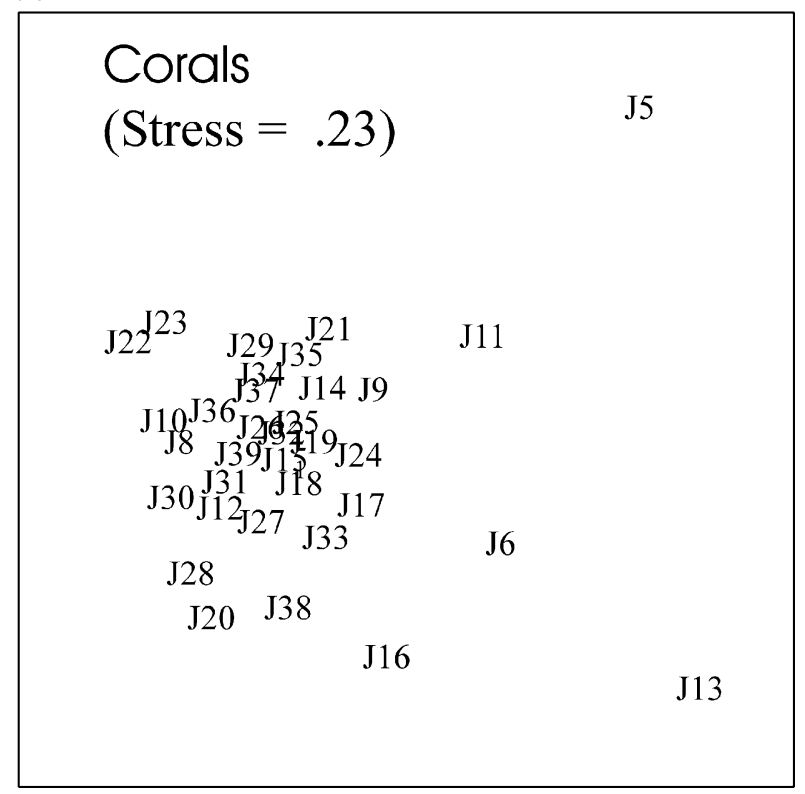

Fig. 1. (a) MDS analysis of fish community structure in Kimbe Bay based on presence-absence of species at 35 sites. (b) MDS analysis of coral community structure in Kimbe Bay based on presence-absence of species at 35 sites.

In general, the accumulation of species in reserves with additional sites was rapid, with $>50 \%$ of the species occurring when less than five of the 35 sites were selected (Figs. 3 and 4). However, once $80 \%$ of the species were included by any method, further increases in protected diversity required a substantial increase in the number of sites because of scattered rarities. In practical terms, if we consider seven sites out of the 35 survey sites $(20 \%)$ as a reasonable and achievable target in terms of the number of protected areas possible in Kimbe Bay, a complementarity approach based on

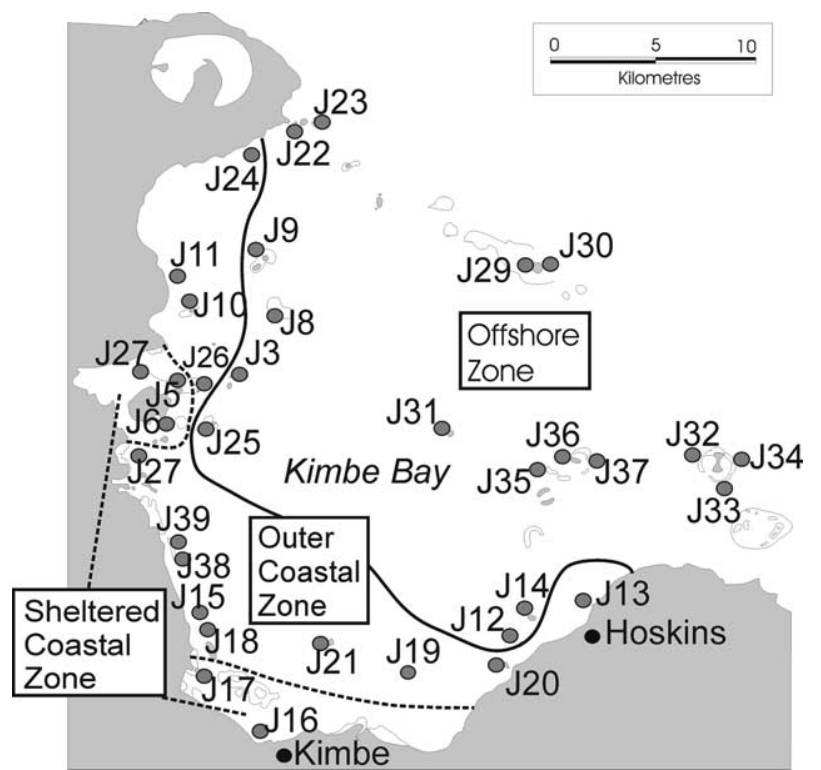

Fig. 2. Sampling sites for fishes and corals in Kimbe Bay, Papua New Guinea, showing the spatial distribution of three eco-habitat zones derived from fish community analysis (see Fig. 1b).

richness would include $76.9 \%$ of fish species, compared with only $63.8 \%$ for a random protocol (Table 2 ). The complementarity and hotspot approaches based on rarity provided the same level of improvement over the random protocol. In order to include all species into a reserve network, 34 sites would be required using complementarity-based and rarity hotspot approaches, and all 35 sites for all other approaches. To include all fish species in protected areas would require 31 sites by complementarity (richness), while all other methods would require all 35 sites. At low numbers of protected sites, there was a distinctly higher proportional inclusion of coral species compared to fishes (Table 2).

Protecting $20 \%$ of the sites would include $84.6 \%$ of corals if based on complementarity and $71.5 \%$ if based on a random model. Total coral species representation can be accomplished by 23 sites for complementarity (rarity), whereas 32 or more sites would be needed for all other selection methods. Clearly, a realistic number of reserve sites will fall short of representing a substantial number of the rarer species for both fishes and corals. For $20 \%$ of sites included in a reserve network, methods that were based on complementarity and hotspot selection (rarity and 3 regions) performed similarly well for fishes and all species, but not for corals alone. The complementarity-based methods clearly outperformed all others for corals (Table 2).

\subsection{Cross taxon congruence}

In order to assess cross-taxon congruence between fishes and corals, we compared the distribution and coincidence of (1) species richness hotspots between 
(a)

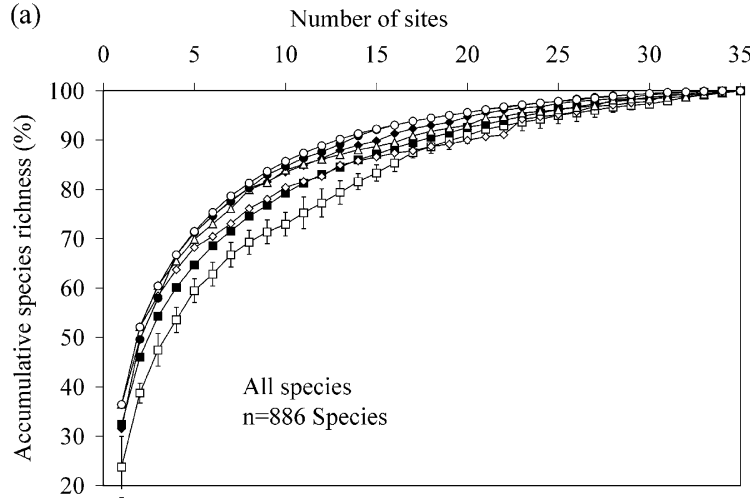

(c)

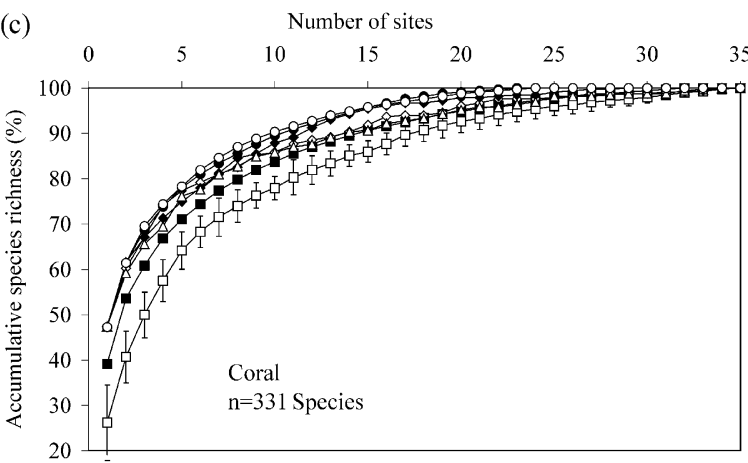

(b)
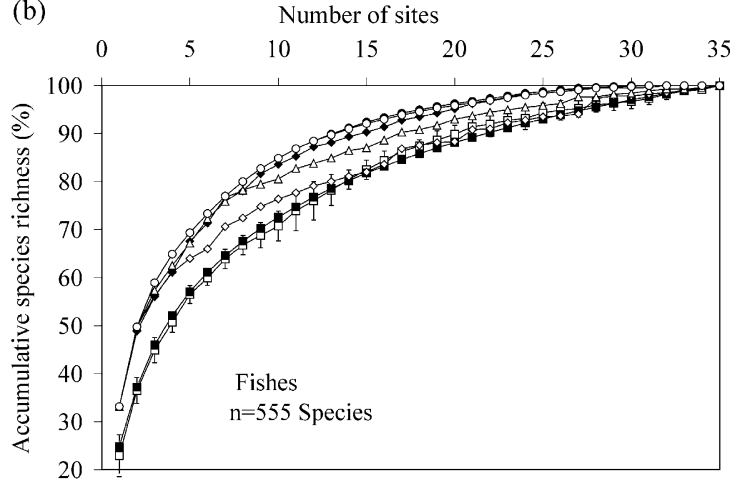

- Random (n=6)

$\diamond$ Hotspots (Richness)

- Hotspots (Rarity)

$\triangleleft$ Hotspots ( 3 regions)

- Complementarity (Rarity)

-o- Complementarity (Richness)

Fig. 3. Species-site accumulation curves for six selection approaches: complementarity (species richness and rarity), hotspots (species richness and rarity), three eco-habitats hotspots and random $(n=6)$. (a) All species, (b) fishes, (c) corals.

corals and fishes and (2) the occurrence of rare species at diversity hotspots. There was no evidence that hotspots in species richness were significantly correlated between fishes and coral species numbers (Pearson correlation coefficient $r=0.290, P>0.05)$, indicating that coral-rich sites do not necessarily harbour a maximum of fish species and vice versa. Fishes and corals also exhibited a different relationship between the distribution of rare species and richness per site. Sites with a high fish species richness do not contain many rare species (Threshold $T=2, r=0.278, P>0.05$ ). In contrast, there was a significant relationship between the occurrence of rare corals and coral richness hotspots $(T=2, r=0.574, P<0.01)$. This indicates that both groups are unevenly distributed across the bay.

There was an asymmetry between fishes and corals in the ability of these taxa to serve as an indicator of the species richness of the other (Fig. 5). There is a distinctly lower degree of representation of fishes or all species if sites are selected based on corals (Fig. 5a). Twenty percent of the sites chosen on the basis of coral richness would select $84.6 \%$ of coral species for protection, but only $68.5 \%$ of fish species and $74.5 \%$ of all species included in the study. Conversely, choice of sites based on fishes produces very similar rates of accumulation of coral species in reserve areas (Fig. 5b). Twenty percent of the sites chosen on this basis would protect $76.9 \%$ of the fishes and $73.8 \%$ of the corals.

\section{Discussion}

\subsection{Selection methods}

The selection of sites in Kimbe Bay based on species richness and scarcity has shown that methods proven to represent a greater number of species in terrestrial environments can also be applied to coral reef systems. All selection procedures significantly improved the representation of species in protected areas in comparison to a random accumulation of protected sites.

Complementarity-based methods for both richness and rarity out-performed all other approaches, confirming patterns described for terrestrial species (Williams et al., 1996; Howard et al., 1998). Previous studies found that the complementarity method based on rare species can be more efficient in capturing biotic diversity than the method based on species richness when there is a large number of rare species present (Pressey et al., 1999). However, in this case the complementarity method based on rarity scored not much better than that based on richness. The greatest disparity between methods occurred over the lower range, in terms of the proportion of sites protected. Thus, selection criteria are likely to make a substantial and important difference for any realistic target level of protection in coral reef systems.

The hotspot methodologies were somewhat intermediate in their performance, lying between choice 


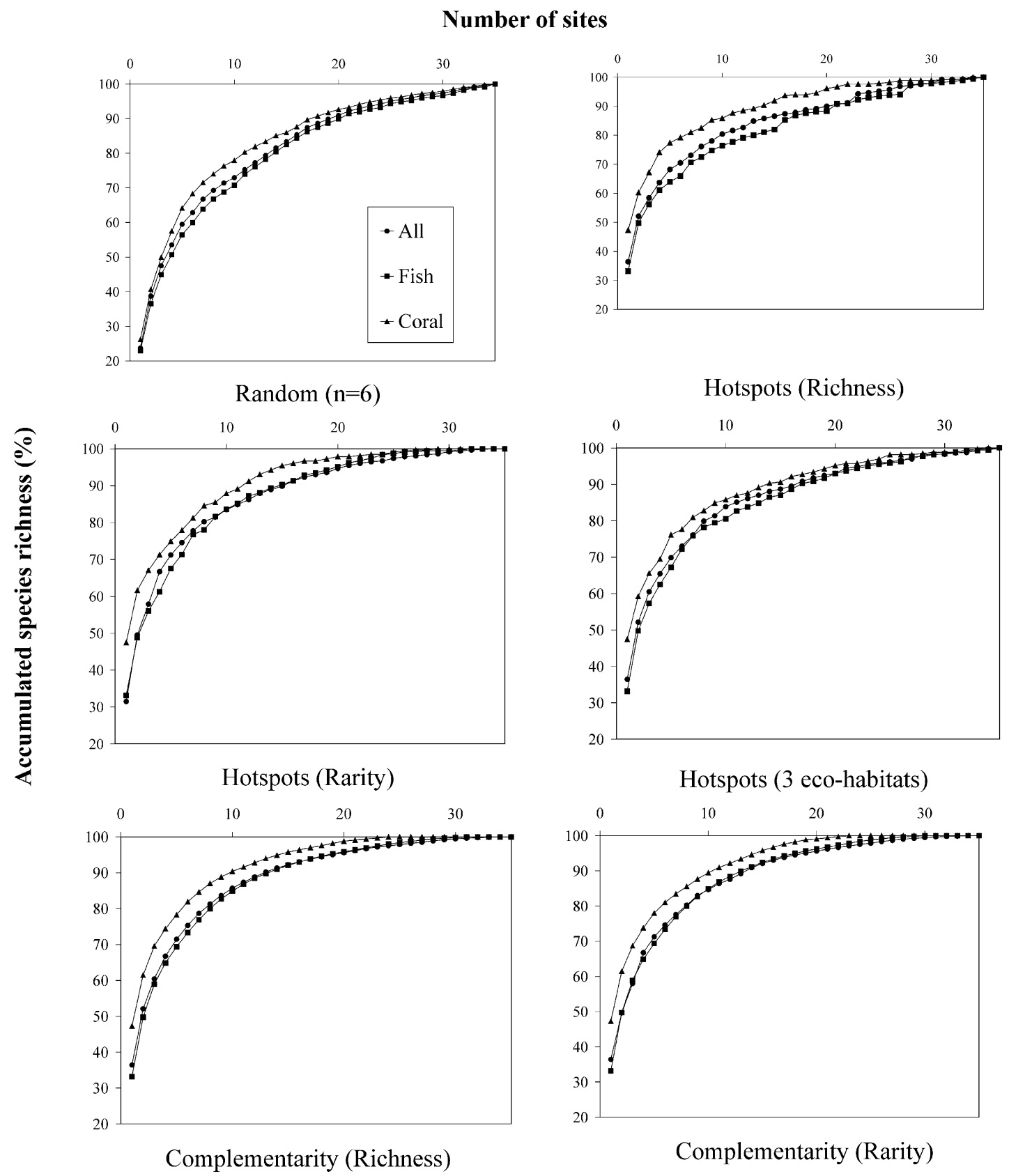

Fig. 4. Site selection for all species, fishes and corals using different selection methods.

based on complementarity and the random model. All methods performed well at low numbers of protected sites and were usually indistinguishable from those for complementarity up to about five protected sites. However, the richness hotspot method was much less efficient in capturing rare species as greater numbers of reserve sites are chosen. The hotspot approach based on rarity hotspots was usually superior to the hotspots methods based on diversity or habitat subdivisions, probably because rare species often occur in sites with low species richness (Prendergast et al., 1993). Although hotspot methods appeared less efficient than complementarity-based approaches, hotspot approaches could be suitable if only a low number of sites were to be protected.

Alternating sites chosen from three eco-habitats of the bay, based on distance off shore and distinct assemblages of fishes, improved the scores of the hotspots (richness) method for fishes only. This reflects the fact that fish communities but not coral communities changed across this gradient. By selecting sites from each region, all fish communities were represented in the first three choices of reserve sites. As a result, this method scores well for top priority sites. In specific settings where only a small number of sites can be selected for protection, and lack of data and expertise call for a 
Table 2

Protection levels in percent achieved by different selection methods when selecting the top seven reserve sites ( $20 \%$ of all 35 sites)

\begin{tabular}{llll}
\hline Selection method & Fish & Coral & All species \\
\hline Complementarity (Richness) & 76.9 & 84.6 & 78.7 \\
Complementarity (Rarity) & 76.9 & 83.4 & 77.5 \\
Hotspots (Richness) & 70.6 & 81.0 & 73.1 \\
Hotspots (Rarity) & 76.7 & 81.3 & 77.7 \\
Hotspots (three eco-habitats) & 75.8 & 80.9 & 76.1 \\
Random & 63.8 & 71.5 & 66.7 \\
\hline
\end{tabular}
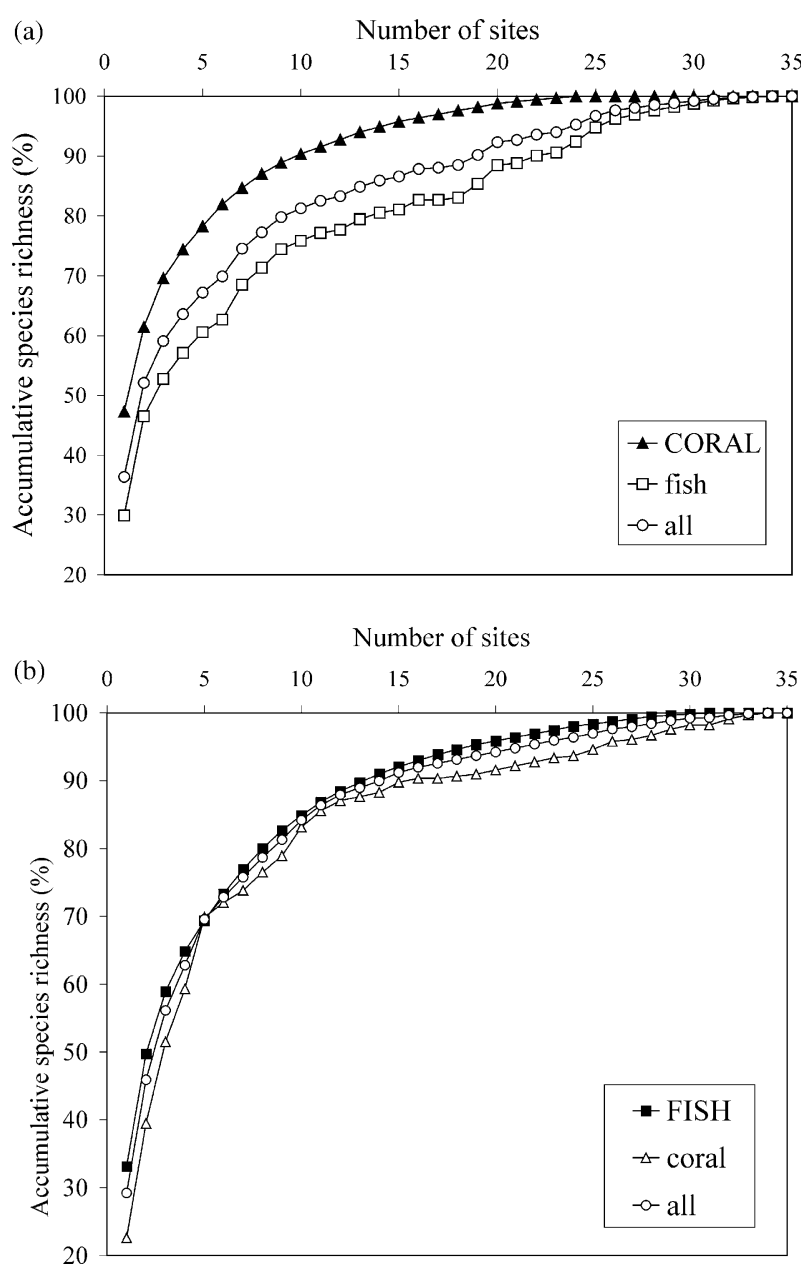

Fig. 5. (a) Accumulation of fish species and all species by site selection based on corals using the complementarity richness method. (b) Accumulation of coral species and all species by site selection based on fishes using the complementarity richness method.

simplistic approach, selecting reserves from each known biogeographical zone or habitat could represent a large proportion of biodiversity. Such an approach might appeal to managers and small local organisations with low resources, as it requires minimal data analysis and expertise (Salm et al., 2000). However, if there is a premium on incorporating rare species, this method has limited value.
The same selection procedures differed in their ability to encompass the biotic diversity of fishes and corals. While there was a distinct advantage of using complementarity-based methods over hotspots methods for corals, fishes and all species, the rarity-based and three eco-habitats hotspot methods were close to the performance of complementarity-based methods. For all methods, higher proportions of all coral species were selected for a given number of reserve sites, when compared to fishes. Corals consistently displayed a steeper slope at the start of the protected species-area curve, reflecting the more homogenous distribution of corals, with a greater proportion of all species represented at any one site. Clearly, there is little correlation between fishes and coral distributions on the scale of the whole bay, and each taxon is responding to different environmental cues. Differences among taxa in distribution patterns and responses to environmental gradients have important implications for the efficacy of different selection procedures.

\subsection{Cross-taxa congruence}

In the literature, cross-taxa congruence established by comparing hotspots coincidence and complementarity scores has been widely used to assess the suitability of taxa as indicator groups in the reserve selection process on a sub-regional scale (Howard et al., 1998; Ward et al., 1999; Moritz et al., 2001; Gladstone, 2002). Groups that display fundamentally similar distribution patterns and biogeography can act as surrogates for overall biodiversity in the terrestrial realm (Prendergast et al., 1993; Gaston, 1996; Williams and Gaston, 1998; Moritz et al., 2001). In Kimbe Bay, however, corals and fishes were not equal in the extent to which they may act as surrogates for one another in the selection of reserve sites. Although there was no significant congruency relationship between hotspots for fishes and corals, selecting sites on the basis of fish biodiversity provided a better representation of coral biodiversity than vice versa, and also represented overall species diversity more efficiently. Similarly, Gladstone (2002) reported that molluscs represent overall species richness better than macroalgae. The better performance of fishes as a surrogate can be explained by their more heterogeneous distribution than corals, and this distribution must be specifically targeted to capture the fish biodiversity. We expect that in general, taxa with homogeneous distributions would be poor surrogates of those with distinct patterns of distributions. This agrees with recent findings in a tropical rainforest, where insects and snails with higher endemism and fine scale distribution performed stronger as surrogates for other taxa (Moritz et al., 2001). However, the situation would be far more complex if reserves were necessary to represent a range of taxa, all with distinct and differing patterns of distribution. 


\subsection{Considerations for reserve selection}

It is important to note that the application of the selection criteria presented here assume that the presence-absence data is accurate for all species, including rare ones (Williams and Gaston, 1994; Rodrigues et al., 2000). Rare species are frequently under-represented when protected areas are selected using species richness hotspots (Prendergast et al., 1993). For coral reef ecosystems, there is little information on rarity and recommendations for the conservation of rare species highlight the need for marine protected areas (Jones et al., 2002). However, the methodologies for collecting coral reef biodiversity data usually involve limited sampling effort at any one site. This is likely to cause an under-estimate of rare species, many of which are cryptic, nocturnal or wide-ranging. The effect of this on the proportion of species represented in reserve sites is unclear. It may lead to an under-estimate of the proportion of species in reserve sites and the diversity of the whole system.

Many factors influence the success of a reserve network in preserving species. While selection procedures based on diversity and rarity are effective for encompassing a large proportion of the species in reserve networks, there are significant limitations to these approaches. Firstly, they do not take into account the likely persistence of species in protected areas (Araujo and Williams, 2000). A species is considered represented when there is only one or a few individuals in a reserve, which is not likely to represent a viable population. In future, selection procedures must take into account the abundance of species and ensure sufficient numbers of individuals of rare species are contained within the same protected area (Nicholls, 1998). This requires information on population size in relation to reef size for a range of species and a better idea of what represents a viable breeding population.

The second problem is that while reserves are the best way to provide some protection for as many species as possible, it is clear that we can never approach a level of protecting $100 \%$ of the species in a region. In Kimbe Bay, the area required to protect all species may approach $80-100 \%$ of the total area. This level of protection will far exceed what is possible in most societies. At most, a target of protecting $20 \%$ of the area may be possible. However, our estimates suggest that this will only cater for $78.7 \%$ of the species, and many of these may be poorly represented in terms of numbers. Allison et al. (1998) have argued that marine reserves are a necessary but not sufficient means to protect exploited populations. We concur with this view. To protect rare species, reserve systems must be coupled with speciesspecific management plans that target rare species of uncertain status. Our study was based on the assumption that selected reserve areas actually receive and sustain protection over a long-term period. In order to sustain such protection and to reduce threats to sites, future efforts should be made to cluster reserves together or improve connectivity between them.

In conclusion, our results support the use of biodiversity-based selection criteria over ad hoc decisions in the establishment of marine conservation areas. We confirm that choosing potential marine reserve sites by complementarity and/or hotspots for rare species will maximise the number of species represented in coral reef reserves. Our comparison of fishes and corals suggests that these techniques achieve different levels of success for different taxa, but a small number of reserve sites $(\sim 20 \%)$ can encompass a large proportion of the extant species of all groups $(78.7 \%)$. When selecting reserves on the basis of a single indicator taxon, care must be taken to choose an indicator that best captures the diversity of other target groups. Although corals are the key habitat-forming organisms on coral reefs, our results suggest that fishes may be more useful surrogates when choosing sites to maximise the protection of both fishes and corals.

\section{Acknowledgements}

We thank The Nature Conservancy who organised the Kimbe Bay survey, G.R. Allen and J. Maragos, who collected fishes and coral data, and Max Benjamin of Walindi Plantation who provided logistic support. We thank P.H. Williams who provided a copy of Worldmap and valuable comments on the manuscript. We thank P.J. Mumby and one anonymous reviewer for their constructive comments. M.B. was supported by the Australian-European Award Programme.

\section{References}

Allen, G.R., Munday, P., 1994. Fish Diversity of Kimbe Bay. The Nature Conservancy, Auckland.

Allison, G.W., Lubchenco, J., Carr, M.H., 1998. Marine reserves are necessary but not sufficient for marine conservation. Ecological Applications 8, 79-92.

Araujo, M.B., Williams, P.H., 2000. Selecting areas for species persistence using occurence data. Biological Conservation 96, 331-345.

Balmford, A., 1998. On hotspots and the use of indicators for reserve selection. Trends in Ecology \& Evolution 10, 409.

Bellwood, D.R., Hughes, T.P., 2001. Regional-scale assembly rules and biodiversity of coral reefs. Science 292, 1532-1534.

Courtney, C.A., White, A.T., 2000. Integrated coastal management in the Philippines: testing new paradigms. Coastal Management 28, 39-53.

Day, J.R., Fernandes, L., Lewis, A., De'ath, G., Slegers, S., Barnett, B., Kerrigan, B., Breen, D., Innes, J., Oliver, J., Ward, T.J., Lowe, D. The representative areas programme. 9th International Coral Reef Symposium, Bali, Indonesia (in press).

Duarte, C.M., 2000. Marine biodiversity and ecosystem services: an elusive link. Journal of Experimental Marine Biology and Ecology $250,117-131$ 
Ferrier, S., Pressey, R.L., Barrett, T.W., 2000. A new predictor of the irreplaceability of areas for achieving a conservation goal, its application to real-world planning, and a research agenda for further refinement. Biological Conservation 93, 303-325.

Gaston, K.J., 1996. Biodiversity-congruence. Progress in Physical Geography 20, 105-112.

Gaston, K.J., Williams, P.H., 1996. Spatial patterns in taxonomic diversity. In: Gaston, K.J. (Ed.), Biodiversity: A Biology of Numbers and Difference. Blackwell Science Ltd, Oxford, pp. 202-229.

Gaston, K.J., Williams, P.H., Eggleton, P., Humphries, C.J., 1995. Large scale patterns of biodiversity - spatial variation in family richness. Proceedings of the Royal Society of London-Series B: Biological Sciences 260, 149-154.

Gladstone, W., 2002. The potential value of indicator groups in the selection of marine reserves. Biological Conservation 104, 211-220.

Hatcher, B.G., Johannes, R.E., Robertson, A.I., 1989. Review of research relevant to the conservation of shallow tropical marine ecosystems. Oceanography and Marine Biology Annual Review 27, 337-414.

Hixon, M.A., Boersma, P.D., Hunter Jr., M.L., Micheli, F., Norse, E.A., Possingham, H.P., Snelgrove, P.V.R., 2001. Oceans at Risk: Research Priorities in Marine Conservation Biology. In: Soule, M.E., Orians, G.H. (Eds.), Conservation Biology: Research Priorities for the Next Decade. Island Press, Covelo, CA.

Holthus, P., 1994. Synthesis report. The Nature Conservancy, Auckland.

Holthus P., Maragos J., 1994. Description of the coral reef habitat. In: TNC., 1994. Kimbe Bay Rapid Ecological Assessment: The Coral Reefs of Kimbe Bay (West New Britain, Papua New Guinea). The Nature Conservancy Auckland

Howard, P.C., Viskanic, P., Davenport, T.R.B., Kigenyi, F.W., Baltzer, M., Dickinson, C.J., Lwanga, J.S., Matthews, R.A., Balmford, A., 1998. Complementarity and the use of indicator groups for reserve selection in Uganda. Nature 394, 472-475.

Jones, G.P., Caley, M.J., Munday, P.I., 2002. Rarity in coral reef fish communities. In: Sale, P.F. (Ed.), Coral Reef Fishes. Dynamics and Diversity in a Complex Ecosystem. Academic Press, San Diego, pp. 81-102.

Jones, G.P., Cole, R.C., Battershill, C.N., 1992. Marine reserves: Do they work? In: Battershill, C.N., Schiel, D.R., Jones, G.P., Creese, R.G., MacDiarmid, A.B. (Eds.), Proceedings of the Second International Temperate Reef Symposium, 7-10 January 1992. NIWA Marine, Wellington, pp. 29-45.

Kirkpatrick, J.B., 1993. An iterative method for establishing priorities for the selection of nature reserves: an example from Tasmania. Biological Conservation 25, 127-134.

Leslie, H., Ruckelshaus, M., Ball, I.R., Andelman, S., Possingham, H.P. Using siting algorithms in the design of marine reserve networks. Ecological Applications (in press).

Maragos, J., 1994. Biodiversity of Stony Corals. The Nature Conservancy, Auckland.

Margules, C.R., Nicholls, A.O., 1988. Selecting networks of reserves to maximise biological diversity. Biological Conservation 48, 63-76.

May, R.M., 1994. Biological diversity, differences between land and sea. Philosophical Transactions Royal Society London B 343, 105-111.

Moritz, C., Richardson, K.S., Ferrier, S., Monteith, G.B., Stanisic, J., Williams, S.E., Whiffin, T., 2001. Biogeographical concordance and efficiency of taxon indicators for establishing conservation priority in a tropical rainforest biota. Proceedings Royal Society London Biological Sciences 268, 1-7.

Munday P.L., 2000. The status of coral reefs in Papua New Guinea. In: GCRMN. Australian Institute for Marine Science, Townsville.

Nicholls, A.O., 1998. Integrating population abundance, dynamics and distribution into broad-scale priority setting. In: Mace, G.M., Balmford, A., Ginssberg, J.R. (Eds.), Conservation in a Changing World. Cambridge University Press, Cambridge.

Prendergast, J.R., Quinn, R.M., Lawton, J.H., 1999. The gaps between theory and practice in selecting nature reserves. Conservation Biology 13, 484-492.
Prendergast, J.R., Quinn, R.M., Lawton, J.H., Eversham, B.C., Gibbons, D.W., 1993. Rare species, the coincidence of diversity hotspots and conservation strategies. Nature 365, 335-337.

Pressey, R.L., Humphries, C.J., Margueles, C.R., Vane-Wright, R.I., Williams, P.H., 1993. Beyond opportunism: key principles for systematic reserve selection. Trends in Ecology and Evolution 8, 124 128.

Pressey, R.L., Possingham, H.P., Logan, V.S., Day, J.R., Williams, P.H., 1999. Effects of data characteristics on the results of reserve selection algorithms. Journal of Biogeography 26, 179-191.

Pressey, R.L., Taffs, K.H., 2001. Scheduling conservation action in production landscapes: priority areas in western New South Wales defined by irreplaceability and vulnerability to vegetation loss. Biological Conservation 100, 355-375.

PRIMER, 2001. Primer 5 for Windows. Available: http://www.primer-e.com.

Reid, W.V., 1998. Biodiversity hotspots. Trends in Ecology and Evolution 13, 275-280.

Reyers, B., van Jaarsveld, A.S., Krueger, M., 2000. Complementarity as a biodiversity indicator strategy. Proceedings Royal Society London Biological Sciences 267, 505-513.

Roberts, C.M., Bohnsack, J.A., Gell, F., Hawkins, J.P., Goodridge, R., 2001. Effects of marine reserves on adjacent fisheries. Science 294, 1920-1923.

Roberts, C.M., Polunin, N.V.C., 1991. Are marine reserves effective in management of reef fisheries? Reviews in Fish Biology and Fisheries $1,65-91$.

Rodrigues, A.S.L., Gaston, K.J., Gregory, R.D., 2000. Using presence-absence data to establish reserve selection procedures that are robust to temporal species turnover. Proceedings of the Royal Society of London-Series B: Biological Sciences 267, 897902.

Salm, R.V., 1984. Ecological boundaries for coral-reef reserves: principles and guidelines. Environmental Conservation 11, 209-215.

Salm, R.V., Clark, J., Siirila, E., 2000. Marine and Coastal Protected Areas: A Guide for Planners and Managers. IUCN, Washington DC.

Steele, J.H., 1985. A comparison of terrestrial and marine ecological systems. Nature 313, 355-358.

Turpie, J.K., Beckley, L.E., Katua, S.M., 2000. Biogeography and the selection of priority areas for conservation of South African coastal fishes. Biological Conservation 92, 59-72.

Vane-Wright, R.I., Humphries, C.J., Williams, P.H., 1991. What to protect? - systematics and the agony of choice. Biological Conservation $55,235-254$.

Ward, T.J., Vanderklift, M.A., Nicholls, A.O., Kenchington, R.A., 1999. Selecting marine reserves using habitats and species assemblages as surrogates for biological diversity. Ecological Applications 9, 691-698.

Werner, T.B., Allen, G.R., 1998. A Rapid Biodiversity Assessment of the Coral Reefs on Milne Bay Province, Papua New Guinea. RAP Working Papers 11. Conservation International, Washington, DC.

Williams, P.H., 2000. WORLDMAP iv WINDOWS: Software and help document 4.2., Privately distributed, London.

Williams, P.H., Burgess, N.D., Rahbek, C., 2000. Flagship species, ecological complementary and conserving the diversity of mammals and birds in sub-Saharan Africa. Animal Conservation 3, 249-260.

Williams, P.H., Gaston, K.J., 1994. Measuring more of biodiversity: can higher-taxon richness predict wholesale species richness? Biological Conservation 67, 211-217.

Williams, P.H., Gaston, K.J., 1998. Biodiversity indicators: graphical techniques, smoothing and searching for what makes relationships work. Ecography 21, 551-560.

Williams, P.H., Gibbons, D., Margules, C.R., Rebelo, A., Humphries, C.J., Pressey, R.L., 1996. A comparison of richness hotspots, rarity hotspots and complementary areas for conserving diversity using British birds. Conservation Biology 10, 155-174. 